\title{
Modeling, Estimation, and Optimization of Equity Portfolios with Heavy-tailed Distributions
}

\author{
Almira Biglova \\ Department of Econometrics, Statistics \\ and Mathematical Finance \\ School of Economics and Business Engineering \\ University of Karlsruhe \\ Kollegium am Schloss, Bau II, 20.12, R210 \\ Postfach 6980, D-76128, Karlsruhe, Germany \\ E-mail: biglovaalmira@mail.ru \\ Sergio Ortobelli \\ Department MSIA \\ University of Bergamo, \\ Via dei Caniana,2 - 24127-Bergamo- Italy \\ E-mail: sergio.ortobelli@unibg.it \\ Svetlozar Rachev \\ Chair of Econometrics, Statistics \\ and Mathematical Finance \\ School of Economics and Business Engineering \\ University of Karlsruhe and Karlsruhe Institute of Technology (KIT) \\ Postfach 6980, 76128 Karlsruhe, Germany and \\ Department of Statistics and Applied Probability \\ University of California, Santa Barbara \\ CA 93106-3110, USA and \\ Finanalytica Inc., Seattle, USA \\ E-mail: rachev@statistik.uni-karlsruhe.de
}

Frank J. Fabozzi ${ }^{1}$

Yale School of Management,

135 Prospect Street, New Haven,

CT 06520-8200, USA,

E-mail: frank.fabozzi@yale.edu

\footnotetext{
${ }^{1}$ Contact Author.

Acknowledgement: We thank Boryana Racheva-Iotova (President, FinAnalytica) and. Stoyan Stoyanov (Chief Quant, FinAnalytica) for helpful comments. Rachev's research was supported by grants from the Division of Mathematical, Life and Physical Science, College of Letters and Science, University of California, Santa Barbara, and the Deutschen Forschungsgemeinschaft. Sergio Ortobelli was partially supported by grants from ex-Murst $40 \%$ 2008 , and 60\% 2008, 2009.
} 


\title{
Modeling, Estimation and Optimization of Equity Portfolios with Heavy-tailed Distributions
}

\begin{abstract}
:
In this chapter we provide a methodology to solve dynamic portfolio strategies considering realistic assumptions regarding the return distribution. First, we analyze the empirical behavior of some equities, suggesting how to approximate an historical return series with a factor model that accounts for most of the variability and proposing a methodology to generate realistic return scenarios. Then we examine the profitability of some reward-risk strategies based on a forecasted evolution of the returns. Since several studies in behavioral finance have shown that most investors in the market are neither risk averters nor risk lovers, we discuss the use of portfolio strategies based on the maximization of performance measures consistent with these investors' preferences. We first argue the computational complexity of reward-risk portfolio selection problems and then we compare the optimal sample paths of the future wealth obtained by performing reward-risk portfolio optimization on simulated data.
\end{abstract}

\section{Introduction}

The purpose of this paper is to model and forecast the behavior of financial asset returns in order to optimize the performance of portfolio choices. In particular, we deal with three fundamental themes in portfolio theory: (1) the reduction of dimensionality of the portfolio problem, (2) the generation of future scenarios, and (3) the maximization of the portfolio performance in a reward-risk plane consistent with investor's behavior. To do so, we suggest a methodology to simulate the joint behavior of future returns to which we apply portfolio selection strategies. For this purpose we consider the recent historical observations of some equities, paying attention to the modeling of all distributional aspects of the financial series. The empirical analysis performed on these series suggest that they exhibit (1) asymmetry and heavy tailedness and (2) volatility clustering such that calm periods are generally followed by highly volatile periods and vice versa. Moreover, the findings suggest that the dependence model has to be flexible enough to account for the asymmetry of central dependence and, even more importantly, dependence of the tail events ("huge losses go together"). It is no surprise that the Gaussian distributional assumption is rejected for the financial series in our study. In fact, these results are largely confirmed by several empirical studies. ${ }^{2}$

In searching for an acceptable model to describe the dependence structure, we first perform a principal components analysis (PCA) to identify the main portfolio factors whose variance is significantly different from zero. By doing so, we obtain the few components that explain the majority of the return volatility, resulting in a reduction of the dependence structure dimension. In order to simulate realistic future return scenarios, we distinguish between the approximation of PCA-residuals and PCA-factors. The sample residuals obtained from the factor model are well approximated with an $\operatorname{ARMA}(1,1)-\operatorname{GARCH}(1,1)$ model with stable innovations. As a result, we suggest simulating them independently by the simulated factors. Next, we examine the behavior of each factor with a time-series process belonging to the ARMA-GARCH family with stable Paretian innovations and we suggest modeling dependencies with an asymmetric Student $t$ copula valued on the innovations of the factors. ${ }^{3}$ By doing so, we take into account the stylized facts observed in financial markets such as clustering of the volatility effect, heavy tails, and skewness. We then separately model the dependence structure between them.

It is well known that the classic mean-variance framework is not consistent with all investors' preferences. According to several studies, any realistic way of optimizing portfolio performance should maximize upside potential outcomes and minimize the downside outcomes.

\footnotetext{
${ }^{2}$ For a summary of studies, see Rachev and Mittnik (2000), Balzer (2001), Rachev et al. (2005), and Rachev et al. (2007)

${ }^{3}$ See Sun et al. (2008) and Biglova et al. (2008).
} 
For this reason, the portfolio literature since about the late 1990s has proposed several alternative approaches to portfolio selection. ${ }^{4}$ In particular, in this chapter we analyze portfolio selection models based on different measures of risk and reward. However, the resulting optimization problems consistent with investors' preferences could present more local optima. Thus we propose solving them using a heuristic for global optimization. ${ }^{5}$ Finally, we compare the ex-post sample paths of the wealth obtained with the maximization of the Sharpe ratio and of the other performance measures applied to simulated returns. ${ }^{6}$

The chapter is organized as follows. In Section 2 we provide a brief empirical analysis of the dataset used in this study. In Section 3 we examine a methodology to build scenarios based on a simulated copula. In Section 4 we provide a comparison among different strategies. Our conclusions are summarized in Section 5.

\section{Empirical Evidence from the Dow Jones Industrial Average Components}

For purposes of our study, we analyze 30 stocks that were components of the Dow Jones Industrial Average (DJIA) on 10/03/2008. ${ }^{7}$ In particular, we investigate the log returns for 1837 daily observations from 6/14/2001 to $10 / 03 / 2008$ for each of the 30 stocks. Central theories in finance and important empirical studies assume that asset returns follow a normal distribution. The justification of this assumption is often cast in terms of its asymptotic approximation. However this can be only a partial justification because the Central Limit Theorem for normalized sums of independent and identically distributed (i.i.d.) random variables determines the domain of attraction of each stable law. ${ }^{8}$ Therefore, it is not surprising that when we consider tests for normality such as the Jarque-Bera and Kolmogorv-Smirnov tests (with a 95\% confidence level) that the null hypothesis of normality for the daily log returns is rejected for 24 of the 30 stocks. However if we test the stable Paretian assumption with a 95\% confidence level employing the Kolmogorv-Smirnov statistic, we have to reject the null hypothesis only for four of the 30 stocks. Moreover, observing the covariation of the last two years of our data (whose time include also the period of the failure of Lehman Brothers), we deduce that the tails of the return distribution should consider (1) asymmetry of dependence and (2) dependence of the tail events. Therefore, the dependence model cannot be approximated with a multivariate normal distribution because it fails to describe both phenomena.

Even from these preliminary tests it is reasonable to conclude that the assumption of i.i.d. returns and conditional homoskedasticity is not the best model to approximate the return evolution of all equities. Since the prices observed in the market involve information on past market movements, we should consider the return distribution conditioned on information contained in past return data, or a more general information set. The class of autoregressive moving average (ARMA) models is a natural candidate for conditioning on the past of a return series. However, the conditional volatility of ARMA models is independent of past realizations while empirical evidence shows that conditional homoskedasticity is often violated in financial data. In particular, we observe volatility clusters on returns series. Such behaviour is captured by autoregressive conditional heteroskedastic models $(\mathrm{ARCH})^{10}$ and their generalization (GARCH

\footnotetext{
${ }^{4}$ See Balzer (2001), Biglova et al. (2004), Rachev et al. (2008), Ortobelli et al. (2009).

${ }^{5}$ See Angelelli and Ortobelli (2009).

${ }^{6}$ See Sharpe (1994), Biglova et al. $(2004,2009)$.

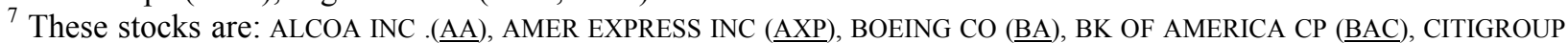
INC (ㄷ), CATERPILLAR INC (CAT), CHEVRON CORP ( $\underline{\text { CVX) }}$, DU PONT E I DE NEM (DD), WALT DISNEY-DISNEY C (DIS), GEN ELECTRIC CO (GE), GEN MOTORS (GM), HOME DEPOT INC (HD), HEWLETT PACKARD CO (HPQ)., IBM, Intel Corporation (INTC), JOHNSON AND JOHNS DC (JNJ), JP MORGAN CHASE CO (JPM), KRAFT FOODS INC (KFT), COCA COLA (KO), MCDONALDS (MCD), 3M COMPANY (MMM), MERCK CO INC (MRK), Microsoft Corporation (MSFT), PFIZER INC (PFE), PROCTER GAMBLE

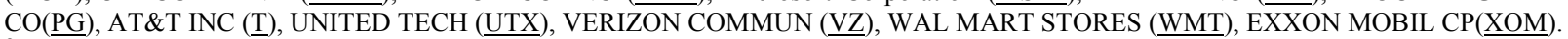

${ }^{8}$ See Zolatorev (1986).

${ }^{9}$ See, for example, Rachev and Mittnik (2000), Rachev et al. (2005), and Rachev et al. (2007).

${ }^{10}$ See Engle (1982).
} 
models), ${ }^{11}$ where the innovations are conditionally stable Paretian distributed. Several empirical experiments by Rachev and Mittnik (2000) have reported the typical behavior problem of time series modeling.

Assume that daily stock returns follow $\operatorname{ARMA}(p, q)-\operatorname{GARCH}(s, u)$ processes; that is, assume

$$
\begin{aligned}
& r_{j, t}=a_{j, 0}+\sum_{i=1}^{p} a_{j, i} r_{j, t-i}+\sum_{i=1}^{q} b_{j, i} \varepsilon_{j, t-i}+\varepsilon_{j, t} \\
& \varepsilon_{j, t}=\sigma_{j, t} z_{j, t} \\
& \sigma_{j, t}^{2}=c_{j, 0}+\sum_{i=1}^{s} c_{j, i} \sigma_{j, t-i}^{2}+\sum_{i=1}^{u} d_{j, i} \varepsilon_{j, t-i}^{2}
\end{aligned},
$$

where $r_{j, t}$ is the daily return of the stock $j(j=1, \ldots, 30)$ at day $t(t=1, \ldots, 1,837)$. Since several studies have shown that ARMA-GARCH filtered residuals are themselves heavy tailed, then it makes sense to assume that the sequence of innovations $z_{j, t}$ is an infinite-variance process consisting of i.i.d. random variables in the domain of normal attraction of a stable distribution with index of stability $\alpha_{j}$ belonging to $(0,2)$. That is, there exist normalizing constants $h_{j}^{(T)} \in R_{+}$ and $k_{j}^{(T)} \in R$ such that

$$
\frac{1}{h_{j}^{(T)}} \sum_{t=1}^{T} z_{j, t}+k_{j}^{(T)} \stackrel{d}{\longrightarrow} S_{\alpha_{j}}\left(\sigma_{j}, \beta_{j}, \mu_{j}\right),
$$

where the constants $h_{j}^{(T)}$ have the form $h_{j}^{(T)}=L_{j}(T) \sqrt[\alpha_{j}]{T}$ and $L_{j}(T)$ are slowly varying functions as $T \rightarrow \infty . S_{\alpha_{j}}\left(\sigma_{j}, \beta_{j}, \mu_{j}\right)$ is a stable Paretian distribution with index of stability, $\alpha_{j} \in(0,2]$, skewness parameter, $\beta_{j} \in[-1,1]$, scale parameter, $\sigma_{j} \in \mathfrak{R}_{+}$, and location parameter, $\mu_{\mathrm{j}} \in \mathfrak{R} .{ }^{12}$ In particular, we can easily test different distributional hypotheses for the innovations of $\operatorname{ARMA}(1,1)-G A R C H(1,1)$ :

$$
\begin{aligned}
& r_{j, t}=a_{j, 0}+a_{j, 1} r_{j, t-1}+b_{j, 1} \varepsilon_{j, t-1}+\varepsilon_{j, t} ; \varepsilon_{j, t}=\sigma_{j, t} z_{j, t} \\
& \sigma_{j, t}^{2}=c_{j, 0}+c_{j, 1} \sigma_{j, t-1}^{2}+d_{j, 1} \varepsilon_{j, t-1}^{2}
\end{aligned}
$$

estimated for the equity returns. First we observe that a simple Ljung-Box Q-statistic for the full model (see Box et al. 1994) indicates that we cannot reject an ARMA(1,1)-GARCH(1,1) model for all return series. Moreover, once the maximum likelihood estimates of the model are obtained from the empirical innovations $\hat{\varepsilon}_{j, t}=r_{j, t}-\hat{a}_{j, 0}+\hat{a}_{j, 1} r_{j, t-1}+\hat{b}_{j, 1} \hat{\varepsilon}_{j, t-1}$, we can easily get the standardized innovations $\hat{z}_{j, t}=\hat{\varepsilon}_{j, t} / \hat{\sigma}_{j, t}$. We can then test these innovations with respect to the stable non-Gaussian distribution versus the Gaussian one by applying the Kolmogorov-Smirnov (KS) statistic according to

$$
K S=\sup _{x \in R}\left|F_{S}(x)-\hat{F}(x)\right|,
$$

where $F_{S}(x)$ is the empirical sample distribution and $\hat{F}(x)$ is the cumulative distribution function evaluated at $x$ for the Gaussian or stable non-Gaussian fit, respectively. The KS test allows a comparison of the empirical cumulative distribution of innovations with either a simulated Gaussian or a simulated stable distribution. For our sample, KS statistics for the stable nonGaussian test is almost 10 times smaller (in average) than the KS distance in the Gaussian case.

\footnotetext{
${ }^{11}$ See Bollerslev (1986).

${ }^{12}$ Refer to Samorodnitsky and Taqqu (1994) and Rachev and Mittnik (2000) for a general discussion of the properties and use of stable distributions.
} 


\section{Generation of scenarios consistent with empirical evidence}

Several problems need to be overcome in order to forecast, control, and model portfolios in volatile markets. First, we have to reduce the dimensionality of the problem, to get robust estimations in a multivariate context. Second, as has been noted in the portfolio selection literature, it is necessary to properly take into consideration the dependence structure of financial returns. Finally, the portfolio selection problem should be based on scenarios that take into account all the characteristics of the stock returns: heavy-tailed distributions, volatility clustering, and non-Gaussian copula dependence.

\subsection{The portfolio dimensionality problem}

When we deal with the portfolio selection problem under uncertainty conditions, we always have to consider the robustness of the estimates necessary to forecast the future evolution of the portfolio. Since we want to compute optimal portfolios with respect to some ordering criteria, we should also consider the sensitivity of risk and reward measures with respect to changes in portfolio composition. Thus, the themes related to robust portfolio theory are essentially twofold: (1) the risk (reward) contribution given by individual stock components of the portfolio ${ }^{13}$ and (2) the estimation of the inputs (i.e., statistical parameters). ${ }^{14}$

As discussed by Rachev et al. (2005) and Sun et al. (2008a, 2008b), the portfolio dimensional problem is strictly linked to the approximation of statistical parameters describing the dependence structure of the returns. Moreover, Kondor et al. (2007) have shown that the sensitivity to estimation error of portfolios optimized under various risk measures can have a strong impact on portfolio optimization, in particular when we consider the probability of rare events. Thus, according to the studies by Papp et al. (2005) and Kondor et al. (2007), robustness of the approximations could be lost if there is not an "adequate" number of observations. In fact, Papp et al. (2005) have shown that the ratio $v$ between the estimated optimal portfolio variance and the true one follows the rule:

$$
v=\left(1-\frac{n}{K}\right)^{-1},
$$

where $K$ is the number of observations and $n$ is the number of assets. Consequently, in order to get a good approximation of the portfolio variance, we need to have a much larger number of observations relative to the number of assets.

Similar results can be proven for other risk parameter estimates such as conditional value at risk. ${ }^{15}$ Because in practice the number of observations is limited, in order to get a good approximation of portfolio input measures, it is necessary to find the right tradeoff between the number of historical observations and a statistical approximation of the historical series depending only on a few parameters.

One way to reduce the dimensionality of the problem is to approximate the return series with a regression-type model (such as a $k$-fund separation model or other model) that depends on an adequate number (not too large) of parameters. ${ }^{16}$ For this purpose, we perform a PCA of the returns of the 30 stocks used in this chapter in order to identify few factors (portfolios) with the highest variability. Therefore, we replace the original $n(n=30$ for our case) correlated time series $r_{i}$ with $n$ uncorrelated time series $P_{i}$ assuming that each $r_{i}$ is a linear combination of the $P_{i}$. Then we implement a dimensionality reduction by choosing only those portfolios whose variance is significantly different from zero. In particular, we call portfolios factors $f_{i}$ the $p$

\footnotetext{
${ }^{13}$ See, for example, Fisher (2003) and Tasche (2000).

${ }^{14}$ See among others, Chopra and Ziemba (1993), Papp et al. (2005), Kondor et al. (2007), Rachev et al. (2005), Sun et al. (2008), and Biglova et al. (2008)).

${ }^{15}$ See Kondor et al. (2007).

${ }^{16}$ See Ross (1978).
} 
portfolios $P_{i}$ with a significant variance, while the remaining $n-p$ portfolios with very small variances are summarized by an error $\varepsilon$. Thus, each series $r_{i}$ is a linear combination of the factors plus a small uncorrelated noise:

$$
r_{i}=\sum_{i=1}^{p} c_{i} f_{i}+\sum_{i=p+1}^{n} d_{i} P_{i}=\sum_{i=1}^{p} c_{i} f_{i}+\varepsilon
$$

Generally, we can apply the PCA either to the variance-covariance matrix or to the correlation matrix. Since returns are heavy-tailed dimensionless quantities, we apply PCA to the correlation matrix obtaining 30 principal components, which are linear combinations of the original series, $r=\left(r_{1}, \ldots, r_{30}\right)^{\prime}$.

Table 1 shows the total variance explained by a growing number of components. Thus, the first component explains $41.2 \%$ of the total variance and the first 14 components explain $80.65 \%$ of the total variance. Because all the other components contribute no more than $1.75 \%$ of the global variance, we implement a dimensionality reduction by choosing only the first 14 factors. As a consequence of this principal component analysis, each series $r_{i}(i=1 \ldots 30)$ can be represented as a linear combination of 14 factors plus a small uncorrelated noise.

\section{TABLE 1 ABOUT HERE}

Once we have identified 14 factors that explain more than $80 \%$ of the global variance, then we can generate the future returns $r_{i}$ using the factor model:

$$
r_{i, t}=\alpha_{i}+\sum_{j=1}^{14} \beta_{i, j} f_{j, t}+e_{i, t} \quad t=1, \ldots, 1837 ; i=1, \ldots, 30
$$

Table 2 reports the coefficients $\alpha_{i} ; \beta_{i, j}$ of factor model (2). The generation of future scenarios should take into account (1) all the anomalies observed in equity returns; (2) the time evolution of factor $f_{j, t}$ and of errors $e_{i, t}$, and; (3) the comovements of the vector of the returns considering the skewness and kurtosis of the joint distribution.

\section{TABLE 2 ABOUT HERE}

To deal with the third problem, we suggest employing a skewed copula with heavy tails. A copula function $C$ associated to random vector $v=\left(v_{1}, \ldots, v_{n}\right)$ is a probability distribution function on the $n$-dimensional hypercube, such that:

$$
F_{v}\left(y_{1}, \ldots, y_{n}\right)=P\left(v_{1} \leq y_{1}, \ldots, v_{n} \leq y_{n}\right)=C\left(P\left(v_{1} \leq y_{1}\right), \ldots, P\left(v_{n} \leq y_{n}\right)\right)=C\left(F_{v_{1}}\left(y_{1}\right), \ldots ., F_{v_{n}}\left(y_{n}\right)\right),
$$

where $F_{v_{i}}$ is the marginal distribution of the $i$-th component (see Sklar (1959)). So once we have generated scenarios with the copula $C\left(u_{1}, \ldots, u_{n}\right)=F_{v}\left(F_{v_{1}}^{-1}\left(u_{1}\right), \ldots, F_{v_{n}}^{-1}\left(u_{n}\right)\right)$ (where $F_{v_{i}}^{-1}$ is the inverse cumulative function of the $i$-th marginal derived from the multivariate distributional assumption $F_{v}$ ) that summarizes the dependence structure of returns, then we can easily generate joint observations using the most opportune inverse distribution functions $\bar{F}_{v_{i}}^{-1}$ of the single components applied to the points generated by the copula. In particular, we next tackle the general problem of return generation considering a multivariate skewed Student's $t$ copula for the joint generation of innovations of the 14 factors.

\subsection{Generation of return scenarios}

Let us summarize the algorithm we propose to generate return scenarios according to the empirical evidence. Assume the log-returns follow model (2). In step 1 of the algorithm we 
approximate each factor $f_{j, t}$ with an $\operatorname{ARMA}(1,1)-\operatorname{GARCH}(1,1)$ process with stable Paretian innovations. Then we provide the marginal distributions for standardized innovations of each factor used to simulate the next-period returns. In the step 2 of the algorithm we estimate the dependence structure of the vector of standardized innovations with a skewed Student $t$. In particular, we first estimate the dependence structure among the innovations with an asymmetric $t$-copula. Then we combine the marginal distributions and the scenarios for the copula into scenarios for the vector of factors. By doing so, we generate the vector of the standardized innovation assuming that the marginal distributions are $\alpha_{j}$-stable distributions and considering an asymmetric $t$-copula to summarize the dependence structure. Then we can easily generate the vector of factors and in the last step of the algorithm we show how to generate future returns.

The algorithm is as follows.

Step 1. Carry out maximum likelihood parameter estimation of $\operatorname{ARMA}(1,1)-\operatorname{GARCH}(1,1)$ for each factor $f_{j, t}(\mathrm{j}=1, \ldots, 14)$

$$
\begin{aligned}
f_{j, t} & =a_{j, 0}+a_{j, 1} f_{j, t-1}+b_{j, 1} \varepsilon_{j, t-1}+\varepsilon_{j, t} \\
\varepsilon_{j, t} & =\sigma_{j, t} u_{j, t} \\
\sigma_{j, t}^{2} & =c_{j, 0}+c_{j, 1} \sigma_{j, t-1}^{2}+d_{j, 1} \varepsilon_{j, t-1}^{2} ; \\
j & =1, \ldots, 14 ; t=1, \ldots, T .
\end{aligned}
$$

Since we have 1,837 historical observations, we use a window of $T=1,837$. Table 3 reports the maximum likelihood estimates for the ARMA-GARCH parameters for all 14 factors.

\section{TABLE 3 ABOUT HERE}

Approximate with $\alpha_{j}$-stable distribution $S_{\alpha_{j}}\left(\sigma_{j}, \beta_{j}, \mu_{j}\right)$ the empirical standardized innovations $\hat{u}_{j, t}=\hat{\varepsilon}_{j, t} / \sigma_{j, t}$ where the innovations $\hat{\varepsilon}_{j, t}=f_{j, t}-a_{j, 0}-a_{j, 1} f_{j, t-1}-b_{j, 1} \varepsilon_{j, t-1} \quad j=1, \ldots, 14 .^{17}$

In order to value the marginal distribution of each innovation, we first simulate $S$ stable distributed scenarios for each of the future standardized innovations series. Then we compute the sample distribution functions of these simulated series:

$$
F_{\hat{u}_{j, T+1}}(x)=\frac{1}{S} \sum_{s=1}^{S} I_{\left\{\hat{u}_{j, T+1}^{(s)} \leq x\right\}}, x \in \square, j=1, \ldots, 14
$$

where $\hat{u}_{j, T+1}^{(s)}(1 \leq s \leq S)$ is the $s$-th value simulated with the fitted $\alpha_{j}$-stable distribution for future standardized innovation (valued in $T+1$ ) of the $j$-th factor.

Step 2. Fit the 14-dimensional vector of empirical standardized innovations $\hat{u}=\left[\hat{u}_{1}, \ldots, \hat{u}_{14}\right]^{\prime}$ with an asymmetric $t$-distribution $\mathbf{V}=\left[V_{1}, \ldots, V_{14}\right]^{\prime}$ with $v$ degree of freedom; that is,

$$
\mathbf{V}=\boldsymbol{\mu}+\boldsymbol{\gamma} Y+\sqrt{Y} \mathbf{Z}
$$

where $\boldsymbol{\mu}$ and $\boldsymbol{\gamma}$ are constant vectors and $Y$ is inverse-gamma distributed $I G(v / 2 ; v / 2)^{18}$ independent of the vector $\mathbf{Z}$ that is normally distributed with zero mean and covariance matrix $\Sigma=\left[\sigma_{i j}\right]$. We use the maximum likelihood method to estimate the parameters $\left(v, \hat{\mu}_{i}, \hat{\sigma}_{i i}, \hat{\gamma}_{i}\right)$ of each component. Then an estimator of matrix $\Sigma$ is given by

$$
\hat{\Sigma}=\left(\operatorname{cov}(\mathbf{V})-\frac{2 v^{2}}{(v-2)^{2}(v-4)} \hat{\gamma} \hat{\gamma}^{\prime}\right) \frac{v-2}{2}
$$

\footnotetext{
${ }^{17}$ For a general discussion on properties and use of stable distributions, see Samorodnitsky and Taqqu (1994) and Rachev and Mittnik (2000).

${ }^{18}$ See among others, Rachev and Mittnik (2000).
} 
where $\hat{\gamma}=\left(\gamma_{1}, \ldots, \gamma_{14}\right)$ and $\operatorname{cov}(\mathbf{V})$ is the variance-covariance matrix of $\mathbf{V}$. Table 4 reports the estimated parameters of the multivariate skewed Student's $t$ distribution for the 14 factors.

\section{TABLE 4 ABOUT HERE}

Since we have estimated all the parameters of $Y$ and $\mathbf{Z}$, we can generate $S$ scenarios for $Y$ and, independently, $S$ scenarios for $\mathbf{Z}$, and using (5) we obtain $S$ scenarios for the vector of standardized innovations $\hat{u}=\left[\hat{u}_{1}, \ldots, \hat{u}_{14}\right]^{\prime}$ that is asymmetric $\boldsymbol{t}$-distributed. Denote these scenarios by $\left(V_{1}^{(s)}, \ldots, V_{14}^{(s)}\right)$ for $s=1, \ldots, S$ and denote the marginal distributions $F_{V_{j}}(x)$ for $1 \leq j \leq 14$ of the estimated 14-dimensional asymmetric $t$-distribution by $F_{V}\left(x_{1}, \ldots, x_{14}\right)=P\left(V_{1} \leq x_{1}, \ldots, V_{14} \leq x_{14}\right)$. Then considering $U_{j}^{(s)}=F_{V_{j}}\left(V_{j}^{(s)}\right), 1 \leq j \leq 14 ; 1 \leq s \leq S$, we can generate $S$ scenarios $\left(U_{1}^{(s)}, \ldots, U_{14}^{(s)}\right)$, $s=1, \ldots, S$ of the uniform random vector $\left(U_{1}, \ldots, U_{14}\right)$ (with support on the 14-dimensional unit cube) and whose distribution is given by the copula

$$
C\left(t_{1}, \ldots, t_{14}\right)=F_{V}\left(F_{V_{1}}^{-1}\left(t_{1}\right), \ldots, F_{V_{14}}^{-1}\left(t_{14}\right)\right) ; 0 \leq t_{i} \leq 1 ; 1 \leq i \leq 14 .
$$

Considering the stable distributed marginal sample distribution function of the $j$-th standardized innovation $F_{\hat{u}_{j, T+1}} ; j=1, \ldots, 14$ (see (4)); and the scenarios $U_{j}^{(s)}$ for $1 \leq j \leq 14 ; 1 \leq s \leq S$, then we can generate $S$ scenarios of the vector of standardized innovations (taking into account the dependence structure of the vector) $u_{T+1}^{(s)}=\left(u_{T+1}^{(1, s)}, \ldots, u_{T+1}^{(14, s)}\right), s=1, \ldots, S$ valued at time $T+1$ assuming

$$
u_{T+1}^{(j, s)}=\left(F_{\hat{u}_{j, T+1}}\right)^{-1}\left(U_{j}^{(s)}\right) ; 1 \leq j \leq 14 ; 1 \leq s \leq S .
$$

Once we have described the multivariate behavior of the standardized innovation at time $T+1$ using relation (3), we can generate $S$ scenarios of the vector of innovation

$$
\varepsilon_{T+1}^{(s)}=\left(\varepsilon_{T+1}^{(1, s)}, \ldots, \varepsilon_{T+1}^{(14, s)}\right)=\left(\sigma_{1, T+1} u_{T+1}^{(1, s)}, \ldots, \sigma_{14, T+1} u_{T+1}^{(14, s)}\right), \quad s=1, \ldots, S,
$$

where $\sigma_{j, T+1}$ are still defined by (3). Thus using relation (3) we can generate $S$ scenarios of the vector of factors $f_{T+1}^{(s)}=\left[f_{1, T+1}^{(s)}, \ldots, f_{14, T+1}^{(s)}\right]$ valued at time $T+1$. Observe that this procedure can always be used to generate a distribution with some given marginals and a given dependence structure. ${ }^{19}$

Step 3. In order to estimate future returns valued at time $T+1$, we first estimate a model ARMA(1,1)-GARCH(1,1) for the residuals of the factor model (2). That is, we consider the empirical residuals

$$
\hat{e}_{i, t}=r_{i, t}-\hat{\alpha}_{i}-\sum_{j=1}^{14} \hat{\beta}_{i, j} f_{j, t}
$$

and then we estimate the parameters $g_{i, 0}, g_{i, 1}, h_{i, 1}, k_{i, 0}, \quad k_{i, 1}, p_{i, 1}$ for all $I=1, \ldots, 30$ of the $\operatorname{ARMA}(1,1)-\mathrm{GARCH}(1,1)$

$$
\begin{aligned}
\hat{e}_{i, t}=g_{i, 0}+g_{i, 1} \hat{e}_{i, t-1}+h_{i, 1} q_{i, t-1}+q_{i, t} \\
q_{i, t}=v_{i, t} z_{i, t} \\
v_{i, t}^{2}=k_{i, 0}+k_{i, 1} v_{i, t-1}^{2}+p_{i, 1} q_{i, t-1}^{2} ; \\
\mathrm{i}=1, \ldots, 30 ; t=1, \ldots, T .
\end{aligned}
$$

\footnotetext{
${ }^{19}$ See, among others, Rachev et al. (2005), Sun et al. (2008), Biglova et al. (2008), and Cherubini et al. (2004) for the definition of some classical copula used in finance literature.
} 
Moreover, as for the factor innovation, we approximate with $\alpha_{j}$-stable distribution $S_{\alpha_{i}}\left(\sigma_{i}, \beta_{i}, \mu_{i}\right)$ for any $i=1, \ldots, 30$ the empirical standardized innovations $\hat{z}_{i, t}=\hat{q}_{i, t} / v_{i, t}$, where the innovations $\hat{q}_{i, t}=\hat{e}_{i, t}-g_{i, 0}-g_{i, 1} \hat{e}_{i, t-1}-h_{i, 1} q_{i, t-1}$. Then we can generate $\boldsymbol{S}$ scenarios $\alpha_{j}$-stable distributed for the standardized innovations $z_{i, T+1}^{(s)} \quad s=1, \ldots, S$ and from (6) we get $S$ possible scenarios for the residuals $e_{i, T+1}^{(s)}=v_{i, T+1} z_{i, T+1}^{(s)} \quad s=1, \ldots, S$. Therefore, combining step 2 with the estimation of future residuals from factor model (2), we get $S$ possible scenarios of returns

$$
r_{i, T+1}^{(s)}=\hat{\alpha}_{i}+\sum_{j=1}^{14} \hat{\beta}_{i, j} f_{j, t}^{(s)}+e_{i, t}^{(s)} \quad s=1, \ldots, S .
$$

The procedure illustrated here permits one to generate $S$ scenarios at time $T+1$ of the vector of returns.

\section{The portfolio selection problem}

Suppose we have a frictionless market in which no short selling is allowed and all investors act as price takers. The classical portfolio selection problem among $n$ assets in the reward-risk plane consists of minimizing a given risk measure $\rho$ provided that the expected reward $v$ is constrained by some minimal value $m$; that is,

$$
\begin{gathered}
\min _{x} \rho\left(x^{\prime} r-r_{b}\right) \\
\text { s.t. } \\
v\left(x^{\prime} r-r_{b}\right) \geq m ; x_{i} \geq 0, \sum_{i=1}^{n} x_{i}=1 ;
\end{gathered}
$$

where $r_{b}$ denotes the return of a given benchmark, and $x^{\prime} r=\sum_{i=1}^{n} x_{i} r_{i}$ stands for the returns of a portfolio with composition $x=\left(x_{1}, \ldots, x_{n}\right)^{\prime}$. The portfolio that provides the maximum expected reward $v$ per unit of risk $\rho$ is called the market portfolio and is obtained from problem (8) for one value $m$ among all admissible portfolios. In particular, when the reward and risk are both positive measures, the market portfolio is obtained as the solution of the optimization problem

$$
\begin{gathered}
\max _{x} \frac{v\left(x^{\prime} r-r_{b}\right)}{\rho\left(x^{\prime} r-r_{b}\right)} \\
\text { s.t. } \\
x_{i} \geq 0, \sum_{i=1}^{n} x_{i}=1
\end{gathered}
$$

Clearly there exist many possible performance ratios $G(X)=\frac{v(X)}{\rho(X)}$.

A first classification with respect to the different characteristics of reward and risk measures is given in Rachev et al. (2008). The most important characteristic is the isotony (consistency) with an order of preference; that is, if $X$ is preferable to $Y$ then $G(X) \geq G(Y) \quad(G(X) \leq G(Y))$. Although the financial literature on investor behavior agrees that investors are non-satiable, there is not a common vision about the investors' aversion to risk. Thus investors' choices should be isotonic with non-satiable investors' preferences (i.e., if $X \geq Y$, then $G(X) \geq G(Y)$ ).

Several behavioral finance studies suggest that most investors are neither risk averse nor risk loving. ${ }^{20}$ Thus, according to Bauerle and Müller (2006)), if risk and reward measures are

\footnotetext{
${ }^{20}$ See Friedman and Savage (1948), Markowitz (1952), Tversky and Kahneman (1992), Levy and Levy (2002), and Ortobelli et al. (2009).
} 
invariant in law (i.e., if $X$ and $Y$ have the same distribution then $\rho(X)=\rho(Y)$ and $v(X)=v(Y)$ ), and the risk measure is positive and convex (concave) and the reward is positive and concave (convex), then the performance ratio is isotone with risk-averse (lover) preferences. Rachev et al. (2008) and Stoyanov et al. (2007) have classified the computational complexity of reward-risk portfolio selection problems. In particular, Stoyanov et al. (2007) have shown that we can distinguish four cases of reward/risk ratios $G(X)$ that admit unique optimum portfolio strategies. The most general case with unique optimum is when the ratio is a quasi-concave function; that is, the risk functional $\rho(X)$ is positive convex and the reward functional $v(X)$ is positive concave. As observed above, by maximizing the ratio $G(X)$ we obtain optimal choices for riskaverse investors. In the other cases, when both measures $\rho(X)$ and $v(X)$ are either concave or convex, then the ratio $G(X)$ is isotone with investors' preferences that are neither risk averse nor risk loving. However, in this last case the performance ratio admits more local optima.

\subsection{Review of Performance Ratios}

Here we will review three performance ratios that we will use in the next section when we perform our empirical comparisons: Sharpe ratio, Rachev ratio, and Rachev higher moments ratio.

According to Markowitz' mean-variance analysis, Sharpe (1994) suggested that investors should maximize what is now referred to as Sharpe ratio (SR) given by

$$
S R\left(x^{\prime} r\right)=\frac{E\left(x^{\prime} r_{T+1}-r_{T+1, b}\right)}{S T D\left(x^{\prime} r_{T+1}-r_{T+1, b}\right)} .
$$

where $S T D\left(x^{\prime} r_{T+1}-r_{T+1, b}\right)$ is the standard deviation of excess returns. Maximizing the Sharpe ratio, we get a market portfolio that should be optimal for non-satiable risk-averse investors, and that is not dominated in the sense of second-order stochastic dominance. The maximization of the Sharpe ratio can be solved as a quadratic-type problem and thus it presents a unique optimum. In contrast to the Sharpe ratio, the next two performance ratios (Rachev ratio and Rachev higher moments ratio) are isotonic with the preferences of non-satiable investors that are neither risk averse nor risk lovers.

The Rachev ratio $(\mathrm{RR})^{21}$ is the ratio between the average of earnings and the mean of losses; that is,

$$
R R\left(x^{\prime} r_{T+1}, \alpha, \beta\right)=\frac{E_{\beta}\left(r_{T+1, b}-x^{\prime} r_{T+1}\right)}{E T L_{\alpha}\left(x^{\prime} r_{T+1}-r_{T+1, b}\right)},
$$

where the ETL is the Expected Tail Loss, also known as Conditional Value-at-Risk (CVaR), is defined as

$$
\operatorname{ETL}_{\alpha}(X)=\frac{1}{\alpha} \int_{0}^{\alpha} \operatorname{VaR}_{q}(X) d q
$$

and

$$
\operatorname{VaR}_{q}(X)=-F_{X}^{-1}(q)=-\inf \{x \mid P(X \leq x)>q\}
$$

is the Value-at-Risk ( $\mathrm{VaR})$ of the random return $X$. If we assume a continuous distribution for the probability law of $X$, then $E T L_{\alpha}(X)=-E\left(X \mid X \leq-\operatorname{VaR}_{\alpha}(X)\right)$ and, therefore ETL, can be interpreted as the average loss beyond VaR. Figure 1 shows the values of this performance ratio when $\alpha=0.01=\beta$ and the components of three assets vary on the simplex

$$
S I M P=\left\{\left(x_{1}, x_{2}, x_{3}\right) \in R^{3} / \sum_{i=1}^{3} x_{i}=1 ; x_{i} \geq 0\right\} .
$$

\footnotetext{
${ }^{21}$ See Biglova et al. (2004).
} 


\section{FIGURE 1 ABOUT HERE}

As we can see from Figure 1, this performance ratio admits more local maxima . In our comparison we consider the parameters $\alpha=0.35$, and $\beta=0.1$.

The Rachev higher moments ratio (RHMR) ${ }^{22}$ is given by

$$
\operatorname{RHMR}\left(x^{\prime} r\right)=\frac{v_{1}\left(x^{\prime} r_{T+1}-r_{T+1, b}\right)}{\rho_{1}\left(x^{\prime} r_{T+1}-r_{T+1, b}\right)}
$$

where

$$
\begin{aligned}
& v_{1}\left(x^{\prime} r-r_{b}\right)=E\left(x^{\prime} r-r_{b} / x^{\prime} r-r_{b}>F_{x^{\prime} r-r_{b}}^{-1}\left(p_{1}\right)\right)+\sum_{i=2}^{4} a_{i} E\left(\left(\frac{x^{\prime} r-r_{b}}{\sigma_{x^{\prime} r-r_{b}}}\right)^{i} \mid x^{\prime} r-r_{b}>F_{x^{\prime} r-r_{b}}^{-1}\left(p_{i}\right)\right) ; \\
& \rho_{1}\left(x^{\prime} r-r_{b}\right)=-E\left(x^{\prime} r-r_{b} / x^{\prime} r-r_{b}<F_{x^{\prime} r-r_{b}}^{-1}\left(q_{1}\right)\right)-\sum_{i=2}^{4} b_{i} E\left(\left(\frac{x^{\prime} r-r_{b}}{\sigma_{x^{\prime} r-r_{b}}}\right)^{i} \mid x^{\prime} r-r_{b}<F_{x^{\prime} r-r_{b}}^{-1}\left(q_{i}\right)\right),
\end{aligned}
$$

$\sigma_{x^{\prime} r-r_{b}}$ is the standard deviation of $x^{\prime} r-r_{b}, a_{i}, \ldots, b_{i} \in \mathrm{R}$ and $p_{i}, q_{i} \in(0,1)$.

This performance ratio was introduced to approximate the non-linearity attitude to risk of decision makers considering the first four moments of the standardized tails of the return distribution. ${ }^{23}$ As we can observe from the definition, the RHMR is very versatile and depends on many parameters. To simplify our analysis in the empirical comparison to follow, we assume $a_{1}=b_{1}=1 ; \quad a_{2}=b_{2}=-1 / 2 ; a_{3}=b_{3}=1 / 6 ; \quad a_{4}=b_{4}=-1 / 24 ; \quad p_{1}=0.9 ; \quad p_{2}=0.89 ; \quad p_{3}=0.88 ;$ $p_{4}=0.87$; and $q_{i}=0.35, i=1,2,3,4$. Figure 2 shows the values of this performance ratio when the composition of three assets varies on the simplex. As we can see from Figure 2, this performance ratio admits more local maxima.

\section{FIGURE 2 ABOUT HERE}

In order to overcome the computational complexity problem for global maximum, we use the heuristic proposed by Angelelli and Ortobelli (2009) that presents significant improvements in terms of objective function and portfolio weights with respect to the classic function fmincon provided with the optimization toolbox of MATLAB. Moreover, this heuristic approximates the global optimum with an error that can be controlled in much less computational time than classic algorithms for global maximum such as simulated annealing.

\subsection{An empirical comparison among portfolio strategies}

In order to value the impact of non-linear reward-risk measures, we provide an empirical comparison among the above strategies based on simulated data. We assume that decision makers invest their wealth in the market portfolio solution given by (9) and we consider the sample path of the final wealth and of the cumulative return obtained from the different approaches. We assume that the investor recalibrates the portfolio daily and has an initial wealth $W_{0}$ equal to 1 and an initial cumulative return $C R_{0}$ equal to 0 (at the date 10/3/2008 when we use $T=1,837$ ). Since we do not know the future evolution of assets returns from $10 / 3 / 2008$, we assume that the returns for each future date correspond to those obtained as the mean of the scenarios and the same for the factors and the residuals of the previous factor model, i.e.,

\footnotetext{
22 See Ortobelli et al. (2009).

${ }^{23}$ See Rachev et al (2008) and Biglova et al (2009).
} 
$r_{i, T+k}=\frac{1}{S} \sum_{s=1}^{S} r_{i, T+k}^{(s)} ; \quad e_{i, T+k}=\frac{1}{S} \sum_{s=1}^{S} e_{i, T+k}^{(s)} \quad$ for $\mathrm{i}=1, \ldots, 30 \quad$ and $\quad f_{j, T+k}=\frac{1}{S} \sum_{s=1}^{S} f_{j, T+k}^{(s)} \quad$ for $\mathrm{j}=1, \ldots, 14$.

Therefore at the $k$-th recalibration, three main steps are performed to compute the ex-post final wealth and cumulative return:

Step 1. Choose a performance ratio. Simulate 3,000 scenarios using the algorithm of the previous section . Determine the market portfolio $x_{M}^{(k)}$ solution to the optimization problem given by (9) that maximizes the performance ratio.

Step 2. The ex-post final wealth is given by:

$$
W_{k+1}=W_{k}\left(\left(x_{M}^{(k)}\right)^{\prime}\left(1+r_{T+k}\right)\right) \text {. }
$$

where $r_{T+k}$ is the vector of returns mean of our scenarios. The ex-post cumulative return is given by:

$$
C R_{k+1}=C R_{k}+\left(x_{M}^{(k)}\right)^{\prime} r_{T+k} .
$$

Step 3. The optimal portfolio $x_{M}^{(k)}$ is the new starting point for the $(k+1)$-th optimization problem given by (9).

Steps 1, 2, and 3 were repeated for all the performance ratios 1,000 times so that we forecasted the future behavior of the optimal portfolio strategies in the next four years. The output of this analysis is represented in Figures 3, 4, and 5. Figure 3 compares the sample paths of wealth and of the total return obtained with the application either of the Angelelli-Ortobelli heuristic or of the local maximization function fmincon of Matlab. This comparison shows that if we maximize the Rachev ratio with $\alpha=0.35 ; \beta=0.1$ with the function for local maximum of Matlab, we could lose more than $20 \%$ of the initial wealth in four years. Figure 4 compares the sample paths of wealth and of the total return obtained with the Rachev ratio and the Sharpe ratio. In particular, the results suggest that using the Rachev ratio we can increase final wealth by more than 25\%. Analogously, Figure 5 shows that using the Rachev Higher Moments ratio we can increase final wealth by more than 15\%. Comparing Figures 4 and 5 we also see the superiority of the Rachev Higher Moments ratio approach relative to the Rachev ratio during the first 300 days. Then we see a superior performance of the Rachev ratio.

What is clear from all of the comparisons is that the use of an adequate statistical and econometric model combined with appropriate risk and performance measures could have a significant impact on the investors' final wealth.

\section{FIGURES 3, 4, AND 5 ABOUT HERE}

\section{Concluding remarks}

In this chapter we provide a methodology to compare dynamic portfolio strategies consistent with the behavior of investors based on realistic simulated scenarios after a reduction of dimensionality of the portfolio selection problem.

We first summarize the empirical evidence regarding the behavior of equity returns: heavy-tailed distributions, volatility clustering, and non-Gaussian copula dependence. Then we discuss how to generate scenarios that take into account the empirical evidence observed for equity return distributions. In particular, we first propose a way to reduce the dimensionality of the problem using principal components analysis. Then we approximate the returns using a factor model on a restricted number of principal components. The factors (i.e., principal components) and residuals of the factor model are modeled with an ARMA(1,1)-GARCH(1,1) with stable innovations. Moreover, we propose a copula approach for the innovations of the factors. This approach allows us to generate future scenarios. Second, we examine the use of reward/risk criteria to select optimal portfolios, suggesting the use of the Sharpe ratio, the Rachev ratio, and the Rachev 
Higher Moments Ratio. Finally, we provide an empirical comparison among final wealth and cumulative return processes obtained using the simulated data. The empirical comparison between the Sharpe ratio and the two Rachev ratios shows the greater predictable capacity of the latter.

\section{References}

Angelelli, E., Ortobelli S. (2009). "American and European portfolio selection strategies: the Markovian approach". To appear as Chapter 5 in Financial Hedging, Novascience, New York.

Balzer, L.A. (2001). "Investment risk: a unified approach to upside and downside returns". In: Sortino F. and Satchell (Eds.) Managing downside risk in financial markets: theory practice and implementation, 103-156. Butterworth-Heinemann, Oxford.

Biglova, A., Kanamura, T., Rachev, S.T., Stoyanov S. (2008). "Modeling,risk assessment and portfolio optimization of energy futures". Investment Management and Financial Innovations 5(1), 17-31.

Biglova, A., Ortobelli, S., Rachev, S., Stoyanov, S. (2009). "A note on the impact of non linear reward and risk measures". To appear in Journal of Applied Functional Analysis.

Biglova, A., Ortobelli, S., Rachev, S., Stoyanov, S. (2004). "Different approaches to risk estimation in portfolio theory". Journal of Portfolio Management 31, 103-112.

Bollerslev, T. (1986). "Generalized autoregressive conditional heteroskedasticity". Journal of Econometrics 31, 307-327.

Box, G.E.P., Jenkins, G.M., Reindel, G.C. (1994). Time Series Analysis: Forecasting and Control. Third edition, Prentice Hall: San Fancisco.

Cherubini,U., Luciano, E., Vecchiato, W. (2004). Copula Methods in Finance. John Wiley \& Sons, Hoboken.

Chopra, C., Ziemba, W. (1993). "The effect of errors in mean variances and covariances on optimal portfolio choice". Journal of Portfolio Management 19, 6-10.

Engle, R.F. (1982). "Autoregressive conditional heteroskedasticity with estimates of the variance of U.K. Inflation". Econometrica 50, 987-1008.

Fischer, T., (2003). "Risk capital allocation by coherent risk measures based on one-sided moments". Insurance: Mathematics and Economics 32 (1), 135-146.

Friedman, M., Savage, L. J.(1948). "The utility analysis of choices involving risk". Journal of Political Economy 56, 279-304.

Kondor, I., Pafka, S., Nagy, G. (2007). "Noise sensitivity of portfolio selection under various risk measures". Journal of Banking and Finance 31, 1545-1573.

Levy, M., Levy, H. (2002). "Prospect theory: much ado about nothing?". Management Science 48, 1334-1349.

Markowitz, H. M. (1952). “The utility of wealth”. Journal of Political Economy 60, 151-158.

Ortobelli, S. Rachev, S., Shalit, H., Fabozzi, F. (2009). "Orderings and probability functionals consistent with preferences". Applied Mathematical Finance 16(1), 81-102

Papp, G., Pafka, S., Nowak, M.A., Kondor, I. (2005). "Random matrix filtering in portfolio optimization". ACTA Physica Polonica B 36, 2757-2765.

Rachev, S., Ortobelli, S., Stoyanov, S., Fabozzi, F. Biglova, A. (2008). "Desirable properties of an ideal risk measure in portfolio theory". International Journal of Theoretical and Applied Finance 11(1), 19-54.

Rachev, S.T., Mittnik, S. (2000). Stable Paretian Models in Finance. John Wiley \& Sons, Chichester.

Rachev,S.T., Mittnik, S., Fabozzi, F.J., Focardi, S.M., Jasic, T. (2007). Financial Econometrics: From Basics to Advanced Modeling Techniques. John Wiley and Sons, New York.

Rachev, S.T., Menn, C., Fabozzi, F.J. (2005). Fat-tailed and Skewed Asset Return Distributions: Implications for Risk Management, Portfolio Selection, and Option Pricing. John Wiley \& Sons, New York. 
Ross, S. (1978). "Mutual fund separation in financial theory-the separating distributions". Journal of Economic Theory 17, 254-286.

Samorodnitsky, G., Taqqu M.S. (1994). Stable Non-Gaussian Random Processes: Stochastic Models with Infinite Variance. CRC Press: New York.

Sharpe, W.F. (1994). "The Sharpe ratio". Journal of Portfolio Management. Fall, 45-58.

Sklar, A. (1959). "Fonctions de Réparitition à n dimensions et Leurs Marges" Publications de l'Institut de Statistique de Universitè de Paris, 8, 229-231.

Stoyanov, S., Rachev, S., Fabozzi, F. (2007). "Optimal financial portfolios". Applied Mathematical Finance 14(5), 401-436.

Sun,W., Rachev,S., Stoyanov,S., Fabozzi F.. (2008). "Multivariate skewed Student's t copula in analysis of nonlinear and asymmetric dependence in German equity market". Studies in Nonlinear Dynamics \& Econometrics, Volume 12(2).

Tasche, D., (2000). "Risk contributions and performance measurement". Working Paper. TU München. http://www-m4.mathematik.tu-muenchen.de/m4/pers/tasche/riskcon.pdf.

Tversky, A. Kahneman, D. (1992). "Advances in prospect theory: Cumulative representation of uncertainty". Journal of Risk and Uncertainty 5, 297-323.

Zolatorev V M (1986). “One-dimensional stable distributions”. Amer. Math. Soc. Transl. of Math. Monographs 65. RI. Transl. of the original 1983 Russian, Providence. 
Table 1. Percentage of the Total Variance Explained by a growing number of components based on the covariance matrix.

\begin{tabular}{|c|c|c|c|c|c|}
\hline $\begin{array}{c}\text { Principal } \\
\text { component }\end{array}$ & $\begin{array}{c}\text { Percentage } \\
\text { of variance } \\
\text { explained }\end{array}$ & $\begin{array}{c}\text { Percentage } \\
\text { of total } \\
\text { variance } \\
\text { explained }\end{array}$ & $\begin{array}{c}\text { Principal } \\
\text { component }\end{array}$ & $\begin{array}{c}\text { Percentage } \\
\text { of variance } \\
\text { explained }\end{array}$ & $\begin{array}{c}\text { Percentage } \\
\text { of total } \\
\text { variance } \\
\text { explained }\end{array}$ \\
\hline 1 & 41.20 & 41.20 & 16 & 1.71 & 84.11 \\
\hline 2 & 5.33 & 46.53 & 17 & 1.64 & 85.75 \\
\hline 3 & 4.52 & 51.05 & 18 & 1.53 & 87.28 \\
\hline 4 & 4.08 & 55.14 & 19 & 1.45 & 88.73 \\
\hline 5 & 3.82 & 58.95 & 20 & 1.40 & 90.13 \\
\hline 6 & 3.24 & 62.19 & 21 & 1.37 & 91.50 \\
\hline 7 & 2.84 & 65.03 & 22 & 1.31 & 92.81 \\
\hline 8 & 2.72 & 67.75 & 23 & 1.24 & 94.05 \\
\hline 9 & 2.63 & 70.38 & 24 & 1.15 & 95.20 \\
\hline 10 & 2.37 & 72.75 & 25 & 1.05 & 96.26 \\
\hline 11 & 2.14 & 74.89 & 26 & 0.97 & 97.23 \\
\hline 12 & 2.03 & 76.92 & 27 & 0.88 & 98.11 \\
\hline 13 & 1.90 & 78.82 & 28 & 0.72 & 98.83 \\
\hline 14 & 1.83 & 80.65 & 29 & 0.65 & 99.48 \\
\hline 15 & 1.75 & 82.40 & 30 & 0.52 & 100.00 \\
\hline
\end{tabular}


Table 2. Estimated Coefficients alpha and betas of the factor model

\begin{tabular}{|c|c|c|c|c|c|c|c|c|c|c|c|c|c|c|c|}
\hline & МММ & & $\mathbf{X P}$ & $\mathbf{T}$ & & BA & & & C & KO & DD & XOM & GE & GM & \\
\hline Inha & & $.017 \%$ & $.004 \%$ & $.010 \%$ & $04 \%$ & $.005 \%$ & $15 \%$ & $012 \%$ & $-0.022 \%$ & $.003 \%$ & $0.004 \%$ & $.013 \%$ & $0.019 \%$ & $0.045 \%$ & $.010 \%$ \\
\hline & & & & & & & & & & & & & & & \\
\hline & & & & & & & & & & & & & & & \\
\hline & & & & & & & & & & & & & $028 \%$ & $146 \%$ & $071 \%$ \\
\hline & & & $121 \%$ & & & & & $89 \%$ & $80 \%$ & $100 \%$ & $37 \%$ & $230 \%$ & $010 \%$ & $.142 \%$ & $331 \%$ \\
\hline eta 5 & & $54 \%$ & $035 \%$ & $310 \%$ & & & & $80 \%$ & $.04 \%$ & $208 \%$ & $90 \%$ & $131 \%$ & $028 \%$ & $52 \%$ & $124 \%$ \\
\hline eta 6 & & & $6 \%$ & $\%$ & & & & & $6 \%$ & $5 \%$ & $2 \%$ & & $55 \%$ & $6 \%$ & \\
\hline beta 7 & & & & & & & & & & & $33 \%$ & $6 \%$ & $13 \%$ & & \\
\hline beta 8 & & & & & & & & & & $333 \%$ & $0.047 \%$ & & $.019 \%$ & $0.018 \%$ & $.120 \%$ \\
\hline & & $058 \%$ & $0.028 \%$ & $0.093 \%$ & $000 \%$ & $114 \%$ & $17 \%$ & $-0.100 \%$ & $003 \%$ & $027 \%$ & $076 \%$ & $052 \%$ & $.078 \%$ & $0.059 \%$ & $0.169 \%$ \\
\hline & & $194 \%$ & $070 \%$ & $-0.020 \%$ & $57 \%$ & $152 \%$ & $06 \%$ & & $067 \%$ & $150 \%$ & $-0.050 \%$ & $007 \%$ & $037 \%$ & $.017 \%$ & $.143 \%$ \\
\hline & & & $10 \%$ & $-0.026 \%$ & $-0.052 \%$ & $-0.327 \%$ & & $-0.102 \%$ & $-0.033 \%$ & $.072 \%$ & $083 \%$ & 0.00070 & $.074 \%$ & $169 \%$ & $.001 \%$ \\
\hline & & $0.069 \%$ & $-0.107 \%$ & $0.026 \%$ & $-0.002 \%$ & $.004 \%$ & $.078 \%$ & $-0.010 \%$ & $-0.052 \%$ & $0.193 \%$ & $.088 \%$ & $-0.004 \%$ & $-0.082 \%$ & $419 \%$ & $.181 \%$ \\
\hline & & $0.058 \%$ & $0.076 \%$ & $0.027 \%$ & $093 \%$ & $015 \%$ & $-0.020 \%$ & $-0.061 \%$ & $-0.010 \%$ & $-0.165 \%$ & $087 \%$ & $-0.040 \%$ & $.017 \%$ & $017 \%$ & $0.051 \%$ \\
\hline \multirow[t]{2}{*}{ beta 14} & & & $010 \%$ & $0.033 \%$ & $68 \%$ & $-0.105 \%$ & $97 \%$ & $05 \%$ & $.072 \%$ & $0.114 \%$ & $131 \%$ & $-0.092 \%$ & $0.025 \%$ & $-0.438 \%$ & $0.272 \%$ \\
\hline & & & TC & NJ & & & & & SFT & & & & & & IS \\
\hline pha & & $33 \%$ & $12 \%$ & $06 \%$ & $1 \%$ & $1 \%$ & $16 \%$ & $18 \%$ & $07 \%$ & $019 \%$ & & $7 \%$ & $12 \%$ & $4 \%$ & $0.001 \%$ \\
\hline ta 1 & & & $670 \%$ & $.434 \%$ & $6 \%$ & $37 \%$ & $4 \%$ & $9 \%$ & $543 \%$ & $0.516 \%$ & $1 \%$ & $44 \%$ & $491 \%$ & $69 \%$ & $0.528 \%$ \\
\hline ta 2 & & $7 \%$ & $56 \%$ & $-0.324 \%$ & $2 \%$ & $88 \%$ & $20 \%$ & $39 \%$ & $24 \%$ & $270 \%$ & $-0.294 \%$ & & $-0.165 \%$ & $14 \%$ & $0.095 \%$ \\
\hline beta 3 & & & $50 \%$ & $-0.048 \%$ & & $35 \%$ & & & & $.079 \%$ & & & $-0.109 \%$ & $93 \%$ & $0.023 \%$ \\
\hline & & & & & & & & & & & & & & & \\
\hline & & & $-0.05 \pi / \%$ & & & & & & & $505 \%$ & & & $-0.281 \%$ & & $-0.023 \%$ \\
\hline & & $-0.043 \%$ & $-0.043 \%$ & $-0.142 \%$ & & & & & $-0.049 \%$ & $-0.281 \%$ & $063 \%$ & $-0.026 \%$ & $179 \%$ & $-0.011 \%$ & $-0.041 \%$ \\
\hline Deta / & & & $0.267 \%$ & $0.025 \%$ & & $050 \%$ & $-0.223 \%$ & & $129 \%$ & $-0.085 \%$ & $.094 \%$ & $32 \%$ & $-0.199 \%$ & $28 \%$ & $0.109 \%$ \\
\hline beta 8 & $9 \%$ & $36 \%$ & $0.086 \%$ & $-0.025 \%$ & $047 \%$ & $399 \%$ & $85 \%$ & $8 \%$ & $-0.002 \%$ & $-0.005 \%$ & $-0.081 \%$ & & $-0.092 \%$ & $-0.167 \%$ & $.031 \%$ \\
\hline a 9 & $66 \%$ & & $-0.045 \%$ & & $9 \%$ & $24 \%$ & $5 \%$ & & $18 \%$ & $-0.002 \%$ & & & $33 \%$ & $3 \%$ & $.057 \%$ \\
\hline 10 & & & $18 \%$ & $45 \%$ & & $16 \%$ & & & $017 \%$ & $-0.051 \%$ & & $4 \%$ & $021 \%$ & $0.205 \%$ & $.087 \%$ \\
\hline & & & $00 \%$ & $48 \%$ & $7 \%$ & $60 \%$ & & $34 \%$ & $-0.101 \%$ & $0.003 \%$ & $0 \%$ & $-0.106 \%$ & $.007 \%$ & $-0.188 \%$ & $.170 \%$ \\
\hline & & & $1560 \%$ & & & & & & & & & & $0.016 \%$ & $-0.022 \%$ & $-0.193 \%$ \\
\hline & & & $0.110 \%$ & & $-0.007 \%$ & & & & & & & & $0.019 \%$ & & $-0.346 \%$ \\
\hline & & $-0.156 \%$ & $-0.104 \%$ & $-0.183 \%$ & $0.100 \%$ & $0.001 \%$ & $0.001 \%$ & & $-0.134 \%$ & & $0.052 \%$ & & & & \\
\hline
\end{tabular}


Table 3. Maximum likelihood estimates of $\operatorname{ARMA}(1,1)-\mathrm{GARCH}(1,1)$ parameters for the 14 factors.

\begin{tabular}{|c|r|r|r|r|r|r|r|}
\hline coefficients & Factor 1 & Factor 2 & Factor 3 & Factor 4 & Factor 5 & Factor 6 & Factor 7 \\
\hline$a_{j, 0}$ & 0.02689 & 0.01643 & 0.01538 & 0.00421 & 0.04306 & 0.02288 & -0.03331 \\
\hline$a_{j, 1}$ & 0.25134 & 0.12943 & -0.20296 & 0.13580 & -0.26676 & -0.35932 & -0.36273 \\
\hline$b_{j, 1}$ & -0.32185 & -0.03155 & 0.25612 & -0.12642 & 0.27020 & 0.40564 & 0.37829 \\
\hline$c_{j, 0}$ & 0.00656 & 0.01864 & 0.01248 & 0.00470 & 0.00000 & 0.17677 & 0.00294 \\
\hline$c_{j, 1}$ & 0.91919 & 0.86903 & 0.90736 & 0.93934 & 0.95628 & 0.71501 & 0.96956 \\
\hline$d_{j, 1}$ & 0.07633 & 0.11862 & 0.07947 & 0.05868 & 0.04372 & 0.10935 & 0.02775 \\
\hline
\end{tabular}

\begin{tabular}{|c|r|r|r|r|r|r|r|}
\hline coefficients & Factor 8 & Factor 9 & Factor10 & Factor11 & Factor12 & Factor13 & Factor14 \\
\hline$a_{j, 0}$ & 0.00197 & -0.01988 & 0.00786 & 0.00034 & 0.00047 & -0.01269 & -0.01689 \\
\hline$a_{j, 1}$ & 0.77222 & -0.60207 & -0.03102 & -0.50587 & 0.94011 & -0.60657 & 0.01424 \\
\hline$b_{j, 1}$ & -0.79557 & 0.64574 & 0.04524 & 0.46606 & -0.93076 & 0.62172 & 0.03502 \\
\hline$c_{j, 0}$ & 0.00428 & 0.00927 & 0.00353 & 0.00665 & 0.01316 & 0.02258 & 0.01895 \\
\hline$c_{j, 1}$ & 0.98033 & 0.95180 & 0.97129 & 0.96058 & 0.94889 & 0.91950 & 0.93939 \\
\hline$d_{j, 1}$ & 0.01504 & 0.03947 & 0.02580 & 0.03321 & 0.03883 & 0.05879 & 0.04197 \\
\hline
\end{tabular}


Table 4. Maximum likelihood estimate of parameters of the skewed Student's $t$ distribution for the 14 factors.

\begin{tabular}{|c|c|c|c|c|c|c|c|c|c|c|c|c|c|c|}
\hline & F1 & F 2 & F 3 & F 4 & F 5 & F 6 & F 7 & F 8 & F 9 & F 10 & F 11 & F 12 & F 13 & F14 \\
\hline$\gamma$ & $-7.19 \%$ & $3.29 \%$ & $-17.5 \%$ & $-4.22 \%$ & $3.95 \%$ & $9.66 \%$ & $2.17 \%$ & $-10.66 \%$ & $1.72 \%$ & $0.03 \%$ & $2.33 \%$ & $-2.68 \%$ & $1.06 \%$ & $-5.87 \%$ \\
\hline $\boldsymbol{\mu}$ & $-2.11 \%$ & $-18.3 \%$ & $39.68 \%$ & $21.77 \%$ & $-9.50 \%$ & $-16.2 \%$ & $7.76 \%$ & $7.56 \%$ & $-6.6 \%$ & $-5.93 \%$ & $-11.9 \%$ & $-9.34 \%$ & $-0.81 \%$ & $19.07 \%$ \\
\hline$v$ & \multicolumn{14}{|c|}{5} \\
\hline \multicolumn{15}{|c|}{ Matrix $\Sigma$} \\
\hline & F1 & F 2 & F 3 & F 4 & F 5 & F 6 & F 7 & F 8 & F 9 & F 10 & F 11 & F 12 & F 13 & F14 \\
\hline 1 & $72.06 \%$ & $47.54 \%$ & $-35.8 \%$ & $-41.1 \%$ & $-28.0 \%$ & $8.48 \%$ & $8.98 \%$ & $11.54 \%$ & $-20.9 \%$ & $6.68 \%$ & $7.11 \%$ & $29.45 \%$ & $9.01 \%$ & $-14.8 \%$ \\
\hline 2 & $47.54 \%$ & $70.38 \%$ & $-33.2 \%$ & $-34.7 \%$ & $-11.3 \%$ & $2.82 \%$ & $16.89 \%$ & $3.54 \%$ & $-10.6 \%$ & $5.87 \%$ & $11.99 \%$ & $23.04 \%$ & $14.53 \%$ & $-23.9 \%$ \\
\hline 3 & $-35.8 \%$ & $-33.2 \%$ & $71.18 \%$ & $39.25 \%$ & $13.87 \%$ & $10.64 \%$ & $-12.5 \%$ & $-3.49 \%$ & $8.46 \%$ & $-15.2 \%$ & $14.68 \%$ & $-14.2 \%$ & $11.15 \%$ & $12.07 \%$ \\
\hline 4 & $-41.1 \%$ & $-34.7 \%$ & $39.25 \%$ & $77.07 \%$ & $17.14 \%$ & $0.25 \%$ & $-2.98 \%$ & $-4.41 \%$ & $3.65 \%$ & $-7.37 \%$ & $-1.15 \%$ & $-24.9 \%$ & $-2.04 \%$ & $13.57 \%$ \\
\hline 6 & $8.48 \%$ & $2.82 \%$ & $10.64 \%$ & $0.25 \%$ & $-1.59 \%$ & $57.56 \%$ & $-1.83 \%$ & $-15.8 \%$ & $5.43 \%$ & $-1.54 \%$ & $6.92 \%$ & $-0.13 \%$ & $13.64 \%$ & $-12.7 \%$ \\
\hline 7 & $8.98 \%$ & $16.89 \%$ & $-12.5 \%$ & $-2.98 \%$ & $-18.1 \%$ & $-1.83 \%$ & $70.42 \%$ & $2.48 \%$ & $-12.5 \%$ & $13.65 \%$ & $-4.07 \%$ & $-13.4 \%$ & $7.58 \%$ & $10.50 \%$ \\
\hline 8 & $11.54 \%$ & $3.54 \%$ & $-3.49 \%$ & $-4.41 \%$ & $-21.0 \%$ & $-15.8 \%$ & $2.48 \%$ & $62.45 \%$ & $-8.60 \%$ & $14.39 \%$ & $-2.50 \%$ & $6.44 \%$ & $-3.28 \%$ & $10.94 \%$ \\
\hline 9 & $-20.9 \%$ & $-10.6 \%$ & $8.46 \%$ & $3.65 \%$ & $10.39 \%$ & $5.43 \%$ & $-12.5 \%$ & $-8.60 \%$ & $61.14 \%$ & $-6.05 \%$ & $-5.60 \%$ & $-14.3 \%$ & $-0.76 \%$ & $6.40 \%$ \\
\hline 10 & $6.68 \%$ & $5.87 \%$ & $-15.2 \%$ & $-7.37 \%$ & $-8.26 \%$ & $-1.54 \%$ & $13.65 \%$ & $14.39 \%$ & $-6.05 \%$ & $70.92 \%$ & $-20.4 \%$ & $-18.4 \%$ & $-0.06 \%$ & $14.32 \%$ \\
\hline 11 & $7.11 \%$ & $11.99 \%$ & $14.68 \%$ & $-1.15 \%$ & $1.86 \%$ & $6.92 \%$ & $-4.07 \%$ & $-2.50 \%$ & $-5.60 \%$ & $-20.4 \%$ & $66.84 \%$ & $28.08 \%$ & $12.74 \%$ & $-15.2 \%$ \\
\hline 12 & $29.45 \%$ & $23.04 \%$ & $-14.1 \%$ & $-24.9 \%$ & $-5.26 \%$ & $-0.13 \%$ & $-13.4 \%$ & $6.44 \%$ & $-14.3 \%$ & $-18.4 \%$ & $28.08 \%$ & $70.98 \%$ & $8.16 \%$ & $-45.0 \%$ \\
\hline 13 & $9.01 \%$ & $14.53 \%$ & $11.15 \%$ & $-2.04 \%$ & $-0.69 \%$ & $13.64 \%$ & $7.58 \%$ & $-3.28 \%$ & $-0.76 \%$ & $-0.06 \%$ & $12.74 \%$ & $8.16 \%$ & $63.76 \%$ & $-15 \%$ \\
\hline 14 & $-14.8 \%$ & $-23.9 \%$ & $12.07 \%$ & $13.57 \%$ & $-14.5 \%$ & $-12.7 \%$ & $10.50 \%$ & $10.94 \%$ & $6.40 \%$ & $14.32 \%$ & $-15.2 \%$ & $-45.0 \%$ & $-15 . \%$ & $70.70 \%$ \\
\hline
\end{tabular}


Figure 1. Rachev ratio with parameters $\alpha=0.01=\beta$ valued varying the composition of three components of DJI

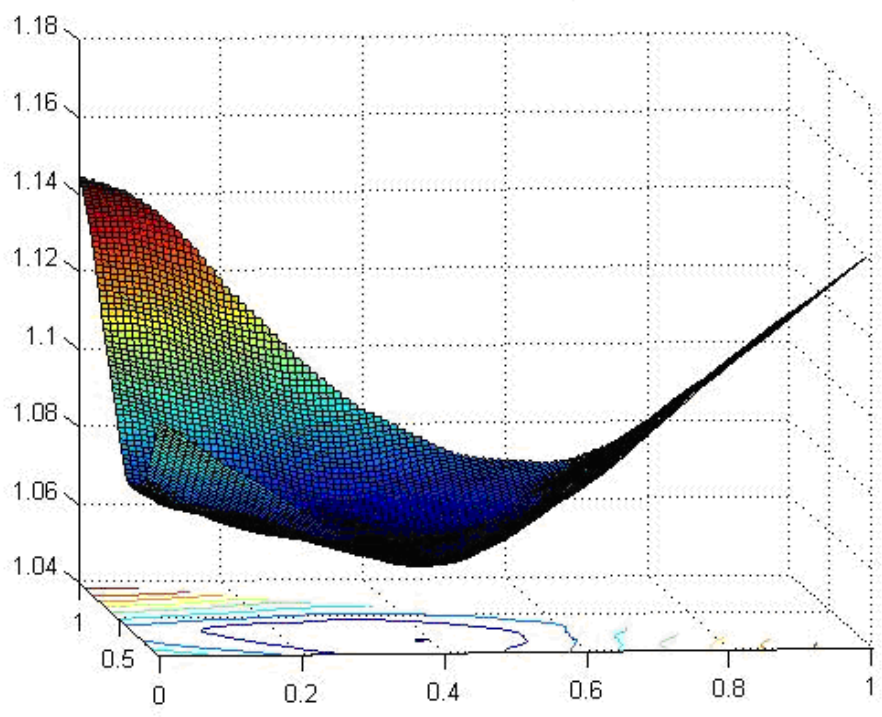


Figure 2. Rachev Higher Moments Ratio Rachev ratio valued varying the composition of three components of DJI

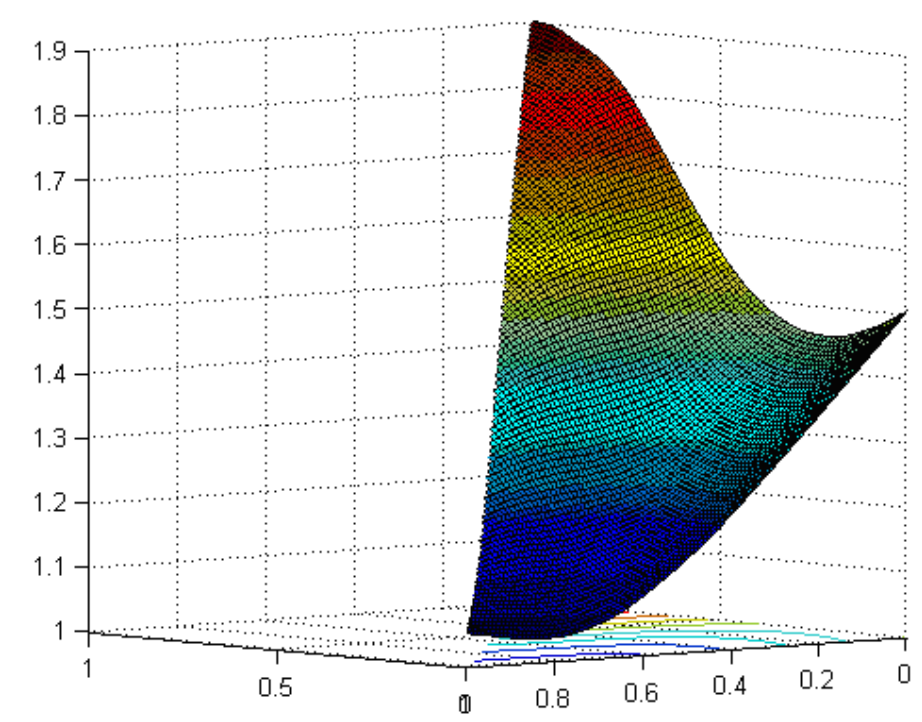


Figure 3. Final wealth and total return realized in 1000 days using the Rachev ratio with parameters $\alpha=0.35 ; \beta=0.1$ and maximizing it either with the Angelelli-Ortobelli heuristic or the function fmincon of Matlab.
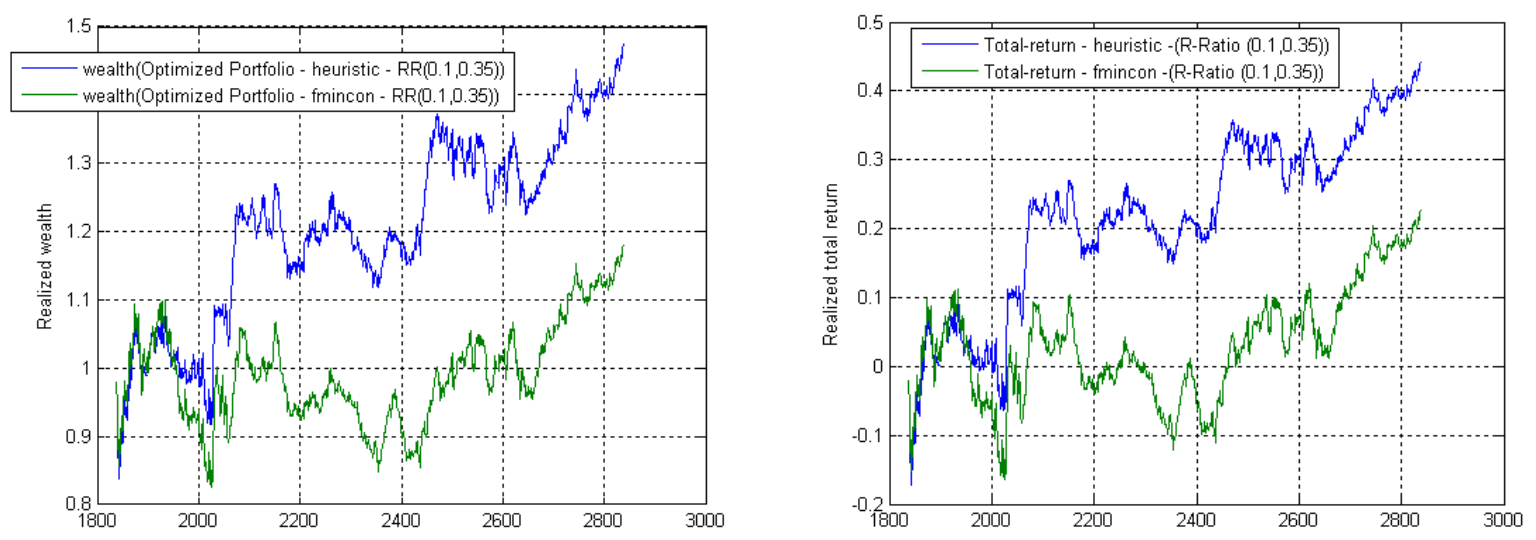
Figure 4. Final wealth and total return realized in 1000 days using either the Rachev ratio with parameters $\alpha=0.35 ; \beta=0.1$ or the Sharpe ratio.
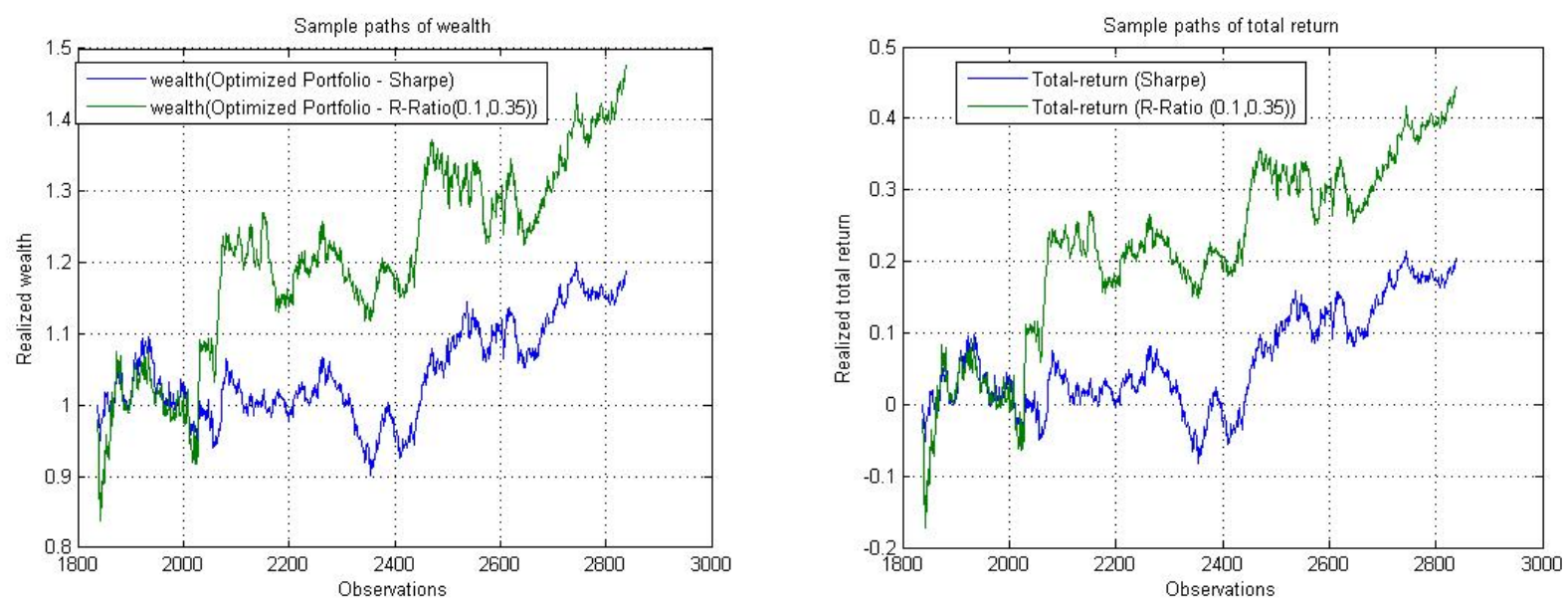
Figure 5. Final wealth and total return realized in 1000 days using either the Rachev high moment ratio or the Sharpe ratio.
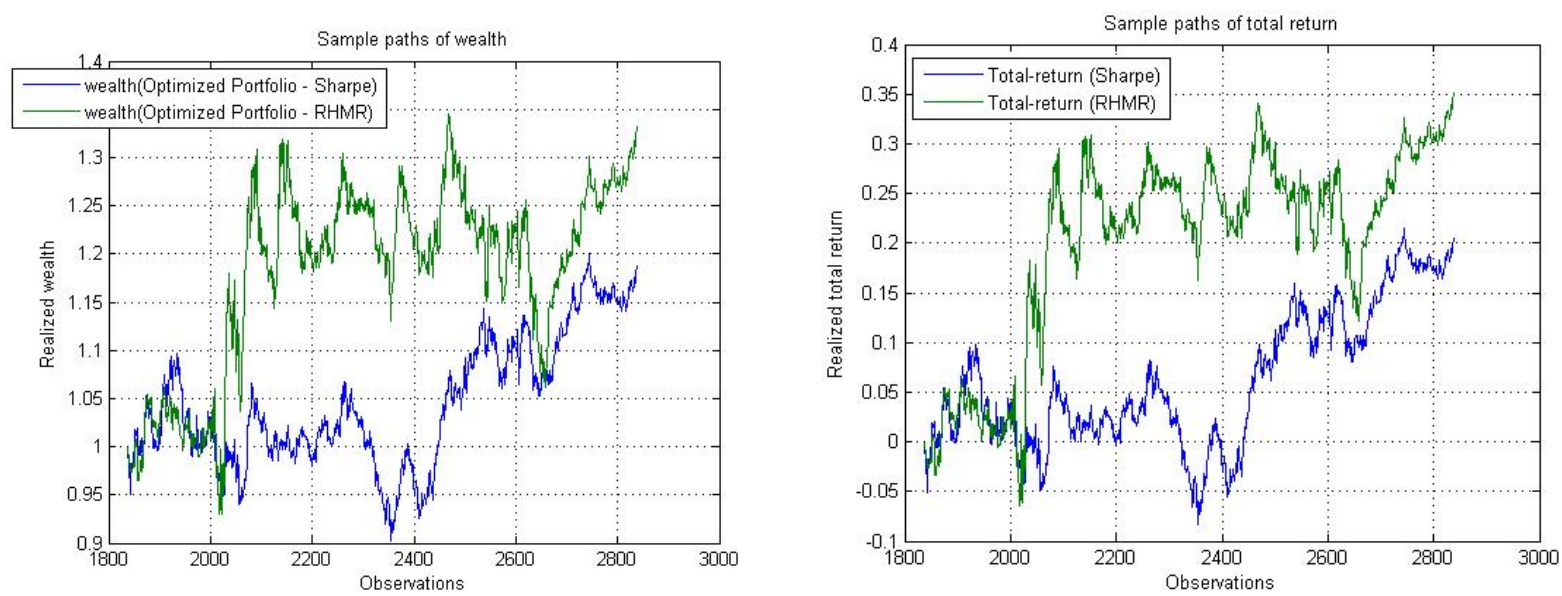